\title{
Lingual Tonsil Lymphatic Tissue Regrowth in Patients Undergoing Transoral Robotic Surgery
}

\author{
Giannicola Iannella, MD (D); Giuseppe Magliulo, MD (D); Filippo Montevecchi, MD; Andrea De Vito, MD, PhD (D); \\ Antonella Polimeni, MD; Marco De Vincentiis, MD; Giuseppe Meccariello, MD; Giovanni D’Agostino, MD; \\ Riccardo Gobbi, MD; Giovanni Cammaroto, MD; Francesco Stomeo, MD; Kenny P. Pang, MD; \\ Brian Rotenberg, MD (D); Claudio Vicini, MD
}

\begin{abstract}
Objectives/Hypothesis: To evaluate a possible regrowth of lingual tonsil lymphatic tissue in patients submitted to lingual tonsil resection with transoral robotic surgery (TORS).

Study Design: Retrospective Study.

Methods: Medical records of patients surgically treated by means of TORS to remove excessive lymphatic tissue of the lingual tonsil were retrospectively reviewed. Postoperative endoscopic data after long-term follow-up were analyzed to investigate possible lymphatic tissue regrowth. Preoperative and postoperative lingual tonsil lymphatic tissue were classified according to the standardized Friedman's grading scale ranging from 0 to 4 .

Results: Sixty-eight patients (41 male and 27 female; mean age $=51.3$ years) were considered suitable for the study analysis. Clinical regrowth was observed in six (8.8\%) patients: four (5.9\%) and two (2.9\%) patients with grade 2 and 3 lymphatic hypertrophy, respectively. No correlation between the grade of regrowth, the time interval from surgery, and the volume of lymphatic tissue removed was found.
\end{abstract}

Conclusions: The lymphatic tissue regrowth after TORS resection appears to be very low.

Key Words: Transoral robotic surgery, lingual tonsil hypertrophy, lymphatic regrowth.

Level of Evidence: 4

Laryngoscope, 00:1-6, 2018

\section{INTRODUCTION}

Lingual tonsil hypertrophy (LTH) is a clinical condition often observed in clinical practice during the inspection of the tongue base region. ${ }^{1-3}$ This lymphatic hypertrophy $(\mathrm{LH})$ is implicated in a variety of airway pathologies, ranging from sleep-related breathing disorders, lingual tonsillitis, and difficult intubation procedures. ${ }^{1-5}$ Additionally, it is frequently a cause of foreign body sensation in the throat and of pharyngodynia in cases of it inflammation. ${ }^{4-6}$

The etiology regarding the growth of this lymphatic tissue (LT) has not been clearly proved. ${ }^{1-6}$ The few studies about this topic showed that previous adenotonsillectomy, laryngopharyngeal reflux (LPR), and a high body

From the Department of Sensory Organs (G.I., G.MAGLIULO, M.D.), and Department of Oral and Maxillofacial Sciences (A.P.), University "Sapienza", Rome, Italy; Otolaryngology-Head and Neck and Oral Surgery Unit (F.M., A.D., G.MECCARIELLO, G.D., R.G., G.C., C.v.), Morgagni Pierantoni Hospital, Forlì, Italy; Department of ENT and Audiology (F.S., C.v.), University of Ferrara, Ferrara, Italy; Department of Otolaryngology, Asia Sleep Centre (K.P.P.), Paragon, Singapore; and the Department of Otolaryngology, Western University (B.R.), London, Ontario, Canada.

Editor's Note: This Manuscript was accepted for publication December 6, 2018.

All authors have made substantial contributions to the conception and design of the study, acquisition of data, data analysis, and interpretation of data. All authors were involved in drafting the manuscript or revising it critically. All authors gave final approval of the version to be published. All authors agreed to be accountable for all aspects of the work.

The authors have no funding, financial relationships, or conflicts of interest to disclose.

Send correspondence to Giannicola Iannella, MD, Via Satrico 7, Rome 00183, Italy. E-mail: giannicolaiannella@hotmail.it

DOI: 10.1002/lary.27775 mass index could be correlated to LTH, even if defined data regarding this aspect are not available. ${ }^{3-7}$

Nowadays, with the advent of transoral robotic surgery (TORS), the removal of this exuberant LT appears easier, and the number of procedures for lingual tonsillectomy have markedly increased. ${ }^{8-12}$ TORS was initially introduced as a surgical technique for the treatment of head and neck cancers. ${ }^{13-16}$ O'Malley et al. ${ }^{13}$ were the first to describe the use of TORS in the treatment of base of tongue (BOT) neoplasms, showing an improved visualization and better instrumental access to the retrolingual space. The TORS role was expanded in 2010 by Vicini et al., ${ }^{17}$ with its application in patients with LH of the BOT and obstructive sleep apnea (OSA) syndrome. Subsequently, several authors reported that the TORS technique allows a safe and effective removal of excessive LT in the BOT region, improving airway obstruction during sleep. ${ }^{8,9,12,18-21}$

Robotic surgery allows blunt dissection of the excessive LT from the muscular plane of the BOT to achieve a complete resection of LT. ${ }^{10,11}$ However, the lingual tonsil LT does not present a capsule dividing it from the underlying tissues, as the palatine tonsils do; therefore, lymphatic cell aggregates may remain after the procedure, especially in the lateral region of the BOT. ${ }^{1-6,9,10,21}$

In patients with LTH who underwent TORS is LT regrowth possible in the long term? In our lengthy experience with TORS, regrowth of the LT was not a frequent observation with the exception of a few cases. The aim of this case series including a review of medical records was 
to evaluate a possible lingual tonsil LT regrowth after robotic resection. Postoperative endoscopic data from patients with marked lymphatic hypertrophy, who underwent TORS in our department, were reviewed after a long-term follow-up to clarify this condition.

\section{MATERIALS AND METHODS}

\section{Study Protocol}

Initially, reports of all TORS procedures consecutively performed at the Otolaryngology-Head and Neck and Oral Surgery Unit of the Morgagni Pierantoni Hospital in Forlì, Italy, between January 2009 and December 2016 were reappraised. Subsequently, the medical records of patients surgically treated using TORS to remove excessive LT of the lingual tonsil were extracted and retrospectively reviewed. All of these patients had a clinical diagnosis of OSA.

All procedures performed in this study involving human participants were in accordance with the ethical standards of the institutional and/or national research committee and with the 1964 Helsinki declaration and its later amendments or comparable ethical standards. Patients were selected for enrollment in this case series according to the following inclusion and exclusion criteria.

\section{Inclusion and Exclusion Criteria}

Patients of all ages were included in the study. The preoperative presence of LTH was the first inclusion criteria. Patients who underwent TORS as oncological treatment were considered not suitable for the study. The absence of LT or the presence of muscular BOT at the preoperative evaluation were considered criteria of exclusion. Patients with an endoscopic follow-up of $<12$ months were excluded from the study, as the clinical followup was considered too short. Finally, patients for whom the preoperative and postoperative data were not collected and patients lost to clinical follow-up were not included in the study.

\section{Evaluation of the BOT LT}

The LT of the lingual tonsil was classified according to the Friedman grading system. ${ }^{1,6,22}$ A standardized grading scale ranging from 0 to 4 (Fig. 1), which can be easily obtained through fiberoptic endoscopy (Table I).,6,22 This grading system is usually executed in our clinical practice, using a flexible fiberoptic endoscope connected to a camera and a high-definition monitor with a recorder. In our department, patients undergoing TORS are usually evaluated with a fiberoptic endoscopy performed at 1 month, 3 months, 6 months, and then annually after surgery. In this study, the data regarding the preoperative endoscopic evaluation (before surgery), the second endoscopic follow-up (endoscopic evaluation at 3 months after surgery), and the final follow-up (last endoscopic evaluation recorded) are reported. Preoperative and postoperative endoscopic recordings were reviewed by the same observer (G.I.) to have homogeneous evaluation of the LT grade.

\section{Notes on Surgical Technique and Intraoperative Findings}

Surgical procedures were performed by different surgeons using the same operatory setting as described by O'Malley et al. ${ }^{13}$ Visualization of the BOT region was obtained with a $30^{\circ}$ up, high-magnification, three-dimensional endoscope. A 5-mm Maryland forceps and a 5-mm monopolar cautery with a spatula tip were used. ${ }^{11}$ The procedure usually requires piecemeal resection in two surgical steps because, generally, the lingual tonsil

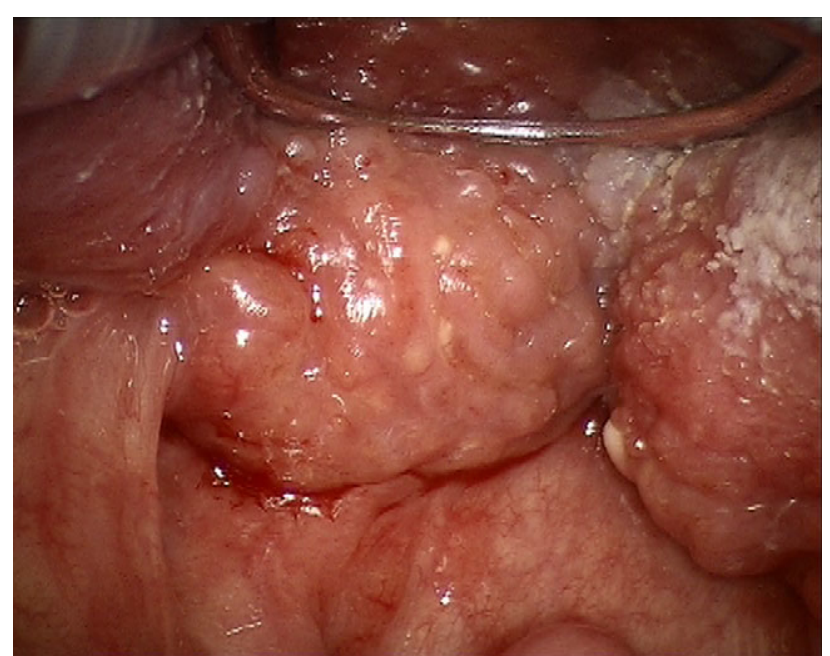

Fig. 1. Transoral robotic surgery procedure. Preoperative grade 4 lymphatic tissue hypertrophy according to the Friedman classification. [Color figure can be viewed in the online issue, which is available at www.laryngoscope.com.]

is composed of two subunits divided by the glossoepiglottic ligament. In this way it is possible to identify and preserve the main anatomic structures of this region. ${ }^{10,11,21}$ The volume (cubic centimeters) of the removed LT was measured in all the procedures using a graduated syringe partially filled with water into which the surgical pieces were placed, thus causing a rise in the level of the water. ${ }^{21}$ All samples were sent for pathologic evaluation after surgical resection to confirm the presence of LTH. Immunohistochemical staining of the samples (CD20, CD79a, CD5, CD30, CD10, bcl2, and bcl6 expression) was performed to rule out lymphoproliferative disorders.

\section{Postoperative Lymphatic Regrowth and Postoperative Surgical Results}

Recurrence was defined as the presence of LT corresponding to a grade 2 to 4 of the Friedman classification at one of the endoscopic follow-ups. A possible correlation between the volume of LT removed and its regrowth has been investigated. Furthermore, LT regrowth has been correlated with the amount of time elapsed since surgery (time in months after surgery). Postoperative symptoms described by the patients of the study at the last follow-up and any pathological conditions found in the BOT region during follow-ups are reported. The difference between preoperative and postoperative apnea-hypopnea index (AHI) values was calculated.

TABLE I.

Friedman Classification of the Lingual Tonsil Lymphoid Tissue. ${ }^{1}$

Grade 0 Complete absence of lymphoid tissue over the tongue base

Grade 1 Lymphoid tissue scattered over the tongue base

Grade 2 Lymphoid tissue covering the entirety of the tongue base with limited vertical thickness

Grade 3 Significantly raised lymphoid tissue covering the entirety of the tongue base, with noticeable vertical thickness approximately 5 to $10 \mathrm{~mm}$ in height

Grade 4 Lymphoid tissue covering the entire tongue base, rising above the tip of the epiglottis, with approximate vertical height $1 \mathrm{~cm}$ or more in thickness 


\section{Statistical Analysis}

The $\chi^{2}$ test was employed to evaluate the significance of the analyzed factors. The Student $t$ test was used to compare preoperative and postoperative results (e.g., LT volume, AHI). Linear regression was used to correlate the Friedman classification score at the last endoscopic follow-up with the volume of the LT removed and the amount of time elapsed after surgery. This research study was performed in accordance with the principles of the Declaration of Helsinki and approved by the local ethics committee.

\section{RESULTS}

A total of 518 TORS procedures were initially reviewed in this case series using medical records. Sixty-eight patients ( 41 male and 27 female, mean age $=51.3$ years, range $=20-75$ years) were considered suitable for the study analysis in accordance with the inclusion and exclusion criteria. All patients suffered from preoperative daytime sleepiness. Foreign body sensation in the throat was reported in $10(25 \%)$ cases. Lymphatic hypertrophy in the region of the BOT was endoscopically evidenced at the preoperative evaluation in all patients: 26 (38.3\%) patients had LTH grade 3 according to the Friedman classification, and $42(61.7 \%)$ a preoperative LTH grade 4 (Table II).

The TORS procedure was well completed in all cases without intraoperative complications. The mean volume of the removed LT was estimated as $11.3 \mathrm{~cm}^{3}$ in the whole series. All patients had a postoperative anatomopathological diagnosis of lymphoid follicular hyperplasia. No cases of lymphoproliferative disorders were observed using immunohistochemical analysis.

At the first fiberoptic endoscopic evaluation considered in this study ( 3 months after surgery), there were $48(70.5 \%)$ patients with a grade 0 and $20(29.5 \%)$ with a grade 1 (small lymphatic cell aggregate scattered in the lateral BOT region) score according to the Friedman classification. Clinical regrowth (Table II) was present in six (8.8\%) patients in the study: four $(5.9 \%)$ and two $(2.9 \%)$ patients with LH grade 2 and 3, respectively. Median and lateral LT regrowth was observed in these cases. No patients with grade 4 regrowth were observed in the study group. The remaining patients were classified as grade 0 in $40(58.8 \%)$ cases and grade 1 in $22(32.4 \%)$. Clinical data of the six patients with LT regrowth are

TABLE II.

Data Regarding the Preoperative and Postoperative Friedman Classification.

\begin{tabular}{lccc}
\hline & $\begin{array}{c}\text { Preoperative } \\
\text { Friedman } \\
\text { Grade }\end{array}$ & $\begin{array}{c}\text { Postoperative Friedman } \\
\text { Grade (3 Months } \\
\text { After Surgery) }\end{array}$ & $\begin{array}{c}\text { Postoperative Friedman } \\
\text { Grade at the } \\
\text { Last Follow-up } \\
\text { (Last Follow-up Obtained } \\
\text { for Each Patient) }\end{array}$ \\
\hline Grade 0 & - & $48(70.5 \%)$ & $40(58.8 \%)$ \\
Grade 1 & - & $20(29.5 \%)$ & $22(32.4 \%)$ \\
Grade 2 & - & - & $4(5.9 \%) *$ \\
Grade 3 & $26(38.3 \%)$ & - & $2(2.9 \%)^{*}$ \\
Grade 4 & $42(61.7 \%)$ & - & - \\
\hline \hline
\end{tabular}

*Percentages of regrowth of the lymphatic tissue (data expressed as number of patients and percentage).

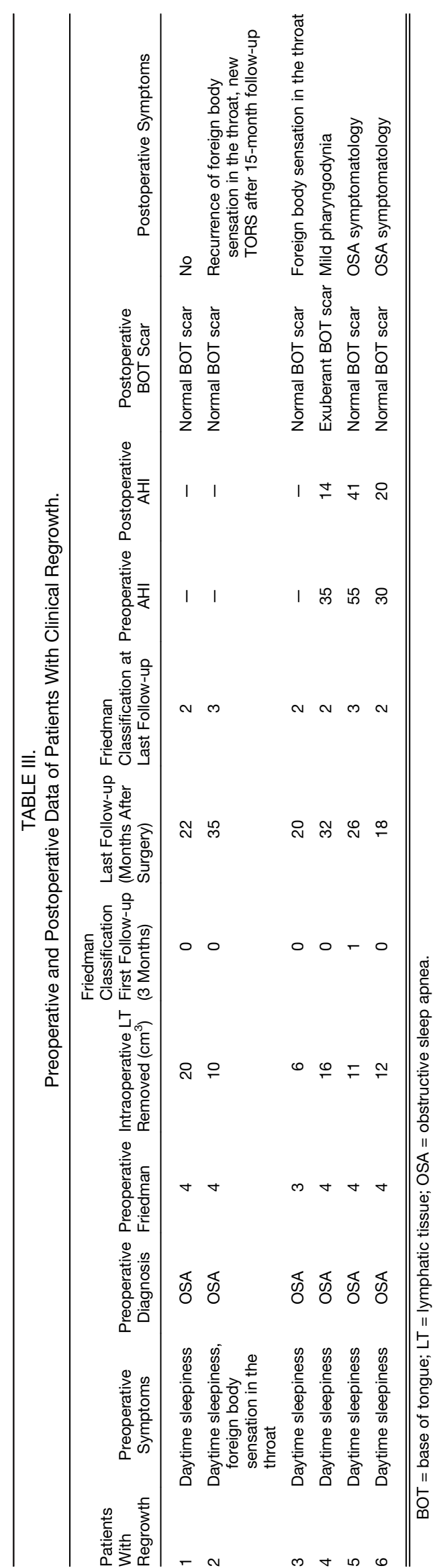

lannella et al.: Lingual Tonsil Lymphatic Tissue Regrowth 


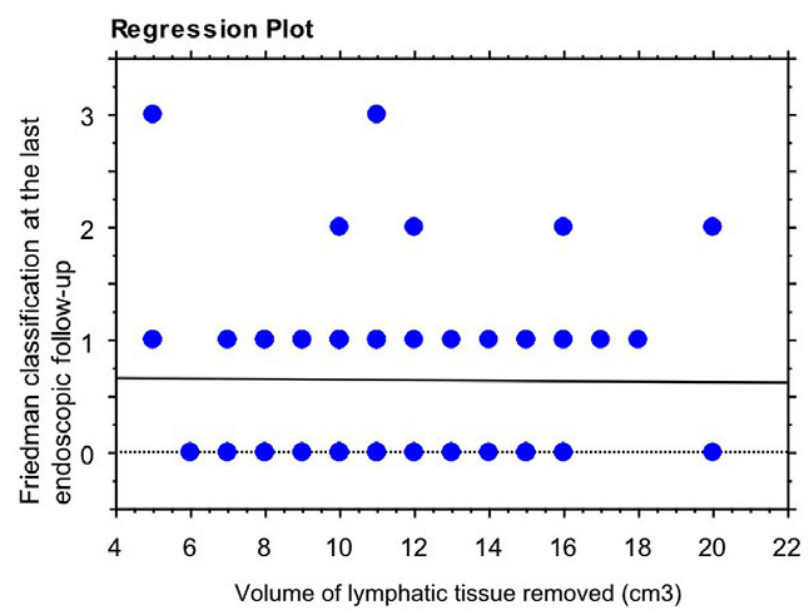

Fig. 2. Linear regression. This is no correlation $(P=.9)$ between volume of lymphatic tissue removed and Friedman grade at the last follow-up. [Color figure can be viewed in the online issue, which is available at www.laryngoscope.com.]

summarized in Table III. All six patients with clinical regrowth were surgically treated by two different surgeons. No differences regarding average age were found between patients with and without LT regrowth $(P=.7)$.

The mean volume of removed LT in the six patients with clinical regrowth was $12.5 \mathrm{~cm}^{3}$. This volume was higher than the mean volume of LT removed in patients for whom clinical regrowth was absent $\left(10.8 \mathrm{~cm}^{3}\right)$. However, no statistical difference was found $(P=.3)$. Moreover, the volume of LT removed did not correlate (regression analysis) with the possible regrowth of LT $(P=.9)$ (Fig. 2).

The mean total time of follow-up (mean of last follow-up recorded for each patients) of the entire study group was 37.5 months (range $=16-92$ months). The mean time interval for LT recurrence, in the six patients who presented with it, was 25.5 months (range $=18-35$ months). The time elapsed since surgery did not correlate with the possibility of LT regrowth $(P=.5)$ (Fig. 3).

A second TORS procedure was performed 15 months after the previous one, in a patient presenting with LT regrowth and recurrence of foreign body sensation of the throat (Fig. 4). In patients without evidence of LT regrowth during the endoscopic follow-ups, few abnormal conditions were observed; two $(2.9 \%)$ cases presented a retention cyst

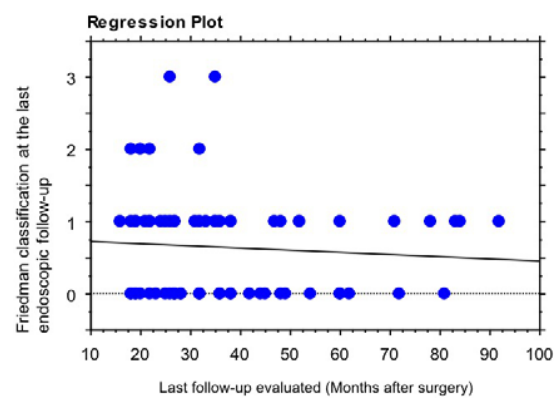

Fig. 3. Linear regression. There is no correlation $(P=.5)$ between the time elapsed since surgery and the Friedman grade at the last follow-up of each patient. [Color figure can be viewed in the online issue, which is available at www.laryngoscope.com.]

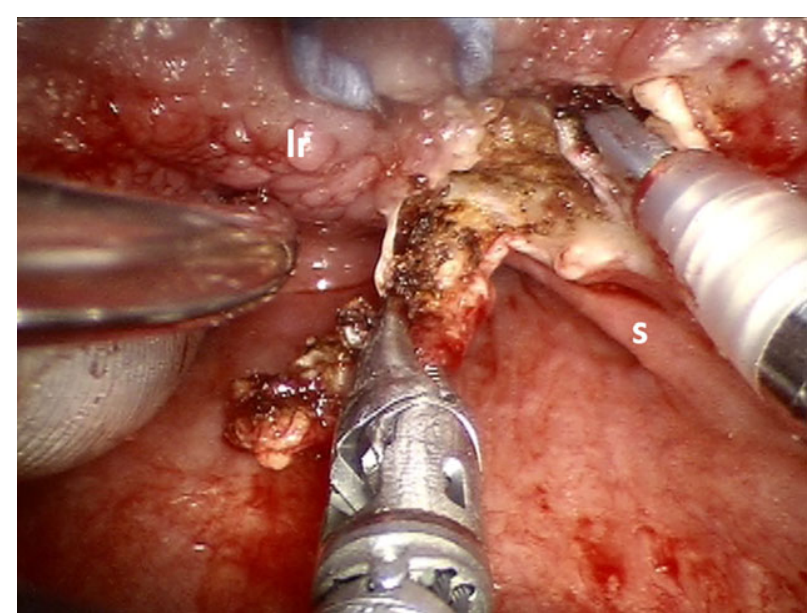

Fig. 4. Second transoral robotic surgery procedure due to lymphatic tissue regrowth (Ir). Visible the scar of the previous robotic lymphatic tissue resection (S). [Color figure can be viewed in the online issue, which is available at www.laryngoscope.com.]

of the region of the BOT, two (2.9\%) patients showed an adhesion between the epiglottis and the BOT region, and another three $(4.4 \%)$ cases presented exuberant scar tissue at the site of the surgical procedure. Three patients complained of recurrent episodes of pharyngodynia, and two reported a persistent foreign body sensation in the throat. The mean preoperative value of AHI went down from 38.2 (95\% confidence interval [CI]: 33.57-42.97) to a a mean value of 21.6 (95\% CI: 16.93-26.33) at the last follow-up. There was a statistical difference between preoperative and postoperative AHI $(P=.001)$.

\section{DISCUSSION}

The lingual tonsil is an immunological organ composed of LT included in Waldeyer's ring. ${ }^{1-6}$ The use of TORS allows LTH resection with good postoperative results. ${ }^{10,11}$ What happens to the resected area over time? Is regrowth of the lingual tonsil LT possible? Nowadays, there is general agreement that this LT could regrow as often happens for similar noncapsulated LT of the adenoids. ${ }^{21-24}$ Although many studies have evaluated postoperative functional results (AHI reduction, pain, swallowing, anatomical reshaping, and quality of life) after TORS BOT resection, ${ }^{8,9,12,18-20}$ no studies have investigated the possibility of long-term regrowth of the LT or the behavior of the lymphatic aggregate remaining after the surgical procedure.

In this study, postoperative data regarding 68 patients with marked lymphatic hypertrophy who submitted to TORS were reviewed after a mean long-term follow-up of 37.5 months to clarify these conditions. All of the patients of this study were preoperatively and postoperatively evaluated according to the Friedman grading system. ${ }^{1}$ This is a standardized grading score proposed by Friedman et al. ${ }^{1}$ to measure LT on the BOT. It represents the most widely used method due to its good cost-effective ratio and its easy application during clinical practice. Furthermore, the systematic use of this classification avoids heterogeneous 
approaches to describe lingual tonsil size/volume. ${ }^{1-6}$ All patients of this study presented a preoperative lymphatic hypertrophy classified as grade $3(38.3 \%)$ or $4(61.7 \%)$ on the Friedman grading system. ${ }^{1}$ This result is in line with other published papers that suggest the possibility of severe grades of LH with TORS. ${ }^{1,6,10,11}$

The etiopathogenetic factors of LTH are not yet clear, and a few studies have been published on this topic. ${ }^{1-5}$ Some studies have shown that LPR could be correlated to $\mathrm{LTH}^{2-6}$ and the patients with Reflux Severity Index scores $>10$ would seem to have a higher incidence of LTH. ${ }^{3}$ In contrast, other authors state that LTH may be due to chronic bacterial infections, immunosuppression, chronic allergies, or a post-tonsillectomy and/or adenoidectomy compensatory growth. ${ }^{1-5}$ Moreover, viral infections (e.g., Epstein-Barr virus, human papillomavirus) could also cause a lymphatic cells proliferation with potential lingual tonsil tissue hypertrophy. ${ }^{3-5}$ Finally, it is our hypothesis that the lymphatic growth could be correlated with vascular mediators or immune-mediated stimulation.

Unfortunately, we could not evaluate the etiopathogenetic factors of LTH, and further studies are underway to analyze the other potential causes of LTH (viral infection, vascular mediators, or immune-mediated stimulation). Clinical LT regrowth was found in $8.8 \%$ patients of the study. These data suggest that lymphatic regrowth is possible but with a very low incidence. Moreover, the grade of regrowth should be considered. In our study, two patients with LT regrowth showed a postoperative grade 3, whereas 4 patients presented with a postoperative grade 2 . Median and lateral LT regrowth was observed in these patients. At the last endoscopic follow-up, no patients showed a grade 4 regrowth, with a remarkable difference between preoperative and postoperative grade 4 incidence (61\% vs. $0 \%$ ). Furthermore, five of the six patients with clinical regrowth were monitored over time and showed stability of LT regrowth, whereas one patient underwent a new TORS procedure after 15 months of follow-up.

The long mean follow-up of the patients analyzed in this study strongly supports our findings. TORS was introduced in our department in $2009,{ }^{17}$ and in this study the mean follow-up was greater than 3 years. Mean follow-up of the patients with LT regrowth was calculated at 25.5 months, and no correlation between the amount of time elapsed since surgery and clinical regrowth emerged $(P=.5)$.

At the last endoscopic follow-up, $22(32.3 \%)$ patients were classified as Friedman grade 1 (small lymphatic cell aggregate scattered in the lateral BOT region); 20 of these patients $(90.1 \%)$ reported a Friedman grade 1 score in the first postoperative endoscopic control. These cases should not be considered as LT regrowth because cell lymphatic aggregates were residual in the lateral area of the BOT after the surgical procedure (incomplete resection). In some cases of massive LTH, it is impossible to completely remove the LT of the BOT to obtain a Friedman score of 0 . It should be considered that lingual tonsil LT does not present a capsule dividing it from the underlying tissues, as palatine tonsils do, and lymphatic cell aggregates may be residual after the procedure, especially in the lateral region of the BOT. ${ }^{1,10,11,21}$ The sparing of lateral LT may also be due to the surgeon's choice of reducing the risk of damaging the lingual artery. $^{11}$

Of the six patients with clear clinical regrowth, only one case presented a small residual lymphatic cell aggregate at the postsurgery first endoscopic follow-up. The other five patients with clinical regrowth did not present postsurgical residual LT. Therefore, our data seem to indicate that $\mathrm{LT}$ regrowth is not related to a residual $\mathrm{LT}$ due to an incomplete resection. The six patients with lymphatic regrowth were surgically treated by two different surgeons, which would seem to indicate that the surgeon's experience is not related to the possibility of regrowth.

Finally, in accordance with the findings reported in other TORS studies. ${ }^{9-12}$, good results in terms of postoperative complications, anatomical function, improvement of preoperative clinical symptoms, and reduction of AHI in OSA patients were found in the present study. Therefore, as claimed by Friedman et al., ${ }^{6}$ robotically assisted resection of the LTH is a validated, effective, and safe technique. TORS offers several advantages, especially regarding visualization of the surgical field compared to other procedures on the base of the tongue (coblation, laser surgery), to achieve a total or near total resection of the LTH with good postoperative results. ${ }^{25,26}$ Further studies are under way to compare TORS with other surgical procedures for removing the LT in the BOT region. The main limitation of this study was its retrospective nature. Further prospective studies regarding lingual tonsil LT regrowth after TORS are warranted.

\section{CONCLUSION}

TORS is a safe and effective technique for performing a total or near total resection of the lingual tonsil hypertrophic tissue. The possibility of LT regrowth after TORS resection is very low. LT regrowth would seem not to be related to the volume of LT removed nor to the amount of time elapsed since surgery.

\section{BIBLIOGRAPHY}

1. Friedman M, Yalamanchali S, Gorelick G, Joseph NJ, Hwang MS. A standardized lingual tonsil grading system: interexaminer agreement. Otolaryngol Head Neck Surg 2015;152:667-672.

2. Tang JA, Friedman M. Incidence of lingual tonsil hypertrophy in adults with and without obstructive sleep apnea. Otolaryngol Head Neck Surg 2018;158:391-394.

3. Hwang MS, Salapatas AM, Yalamanchali S, Joseph NJ, Friedman M. Factors associated with hypertrophy of the lingual tonsils. Otolaryngol Head Neck Surg 2015;152:851-855.

4. Harris MS, Rotenberg BW, Roth K, Sowerby LJ. Factors associated with lingual tonsil hypertrophy in Canadian adults. J Otolaryngol Head Neck Surg 2017;46:32

5. Sung MW, Lee WH, Wee JH, Lee CH, Kim E, Kim JW. Factors associated with hypertrophy of the lingual tonsils in adults with sleep-disordered breathing. JAMA Otolaryngol Head Neck Surg 2013;139:598-603.

6. Friedman M, Wilson MN, Pulver TM, et al. Measurements of adult lingual tonsil tissue in health and disease. Otolaryngol Head Neck Surg 2010;142: $520-525$.

7. DelGaudio JM, Naseri I, Wise JC. Proximal pharyngeal reflux correlates with increasing severity of lingual tonsil hypertrophy. Otolaryngol Head Neck Surg 2008;138:473-478.

8. Miller SC, Nguyen SA, Ong AA, Gillespie MB. Transoral robotic base of tongue reduction for obstructive sleep apnea: a systematic review and metaanalysis. Laryngoscope 2017;127:258-265. 
9. Meccariello G, Cammaroto G, Montevecchi F, et al. Transoral robotic surgery for the management of obstructive sleep apnea: a systematic review and meta-analysis. Eur Arch Otorhinolaryngol 2017;274: $647-653$.

10. Muderris T, Sevil E, Bercin S, Gul F, Kiris M. Transoral robotic lingual tonsillectomy in adults: preliminary results. Acta Otolaryngol 2015;135: 64-69.

11. Vicini C, Montevecchi F, Gobbi R, De Vito A, Meccariello G. Transoral robotic surgery for obstructive sleep apnea syndrome: principles and technique. World J Otorhinolaryngol Head Neck Surg 2017;3:97-100.

12. Justin GA, Chang ET, Camacho M, Brietzke SE. Transoral robotic surgery for obstructive sleep apnea: a systematic review and meta-analysis. Otolaryngol Head Neck Surg 2016;154:835-846.

13. O'Malley BW Jr, Weinstein GS, Snyder W, Hockstein NG. Transoral robotic surgery (TORS) for base of tongue neoplasms. Laryngoscope 2006;116: 1465-1472.

14. Genden EM, Desai S, Sung CK. Transoral robotic surgery for the management of head and neck cancer: a preliminary experience. Head Neck 2009; 31:283-289.

15. Moore EJ, Olsen KD, Kasperbauer JL. Transoral robotic surgery for oropharyngeal squamous cell carcinoma: a prospective study of feasibility and functional outcomes. Laryngoscope 2009;119:2156-2164.

16. Iseli TA, Kulbersh BD, Iseli CE, Carroll WR, Rosenthal EL, Magnuson JS. Functional outcomes after transoral robotic surgery for head and neck cancer. Otolaryngol Head Neck Surg 2009;141:166-171.

17. Vicini C, Dallan I, Canzi P, Frassineti S, La Pietra MG, Montevecchi F. Transoral robotic tongue base resection in obstructive sleep apnoea- hypopnoea syndrome: a preliminary report. ORL J Otorhinolaryngol Relat Spec 2010;72:22-27.

18. Vicini C, Dallan I, Canzi P, et al. Transoral robotic surgery of the tongue base in obstructive sleep Apnea-Hypopnea syndrome: anatomic considerations and clinical experience. Head Neck 2012;34:15-22.

19. Hoff PT, D'Agostino MA, Thaler ER. Transoral robotic surgery in benign diseases including obstructive sleep apnea: safety and feasibility. Laryngoscope 2015;125:1249-1253.

20. Kayhan FT, Kaya KH, Koç AK, et al. Multilevel combined surgery with transoral robotic surgery for obstructive sleep apnea syndrome. J Craniofac Surg 2016;27:1044-1048.

21. Montevecchi F, Cammaroto G, Meccariello G, et al. Trans-oral robotic surgery (TORS) for the treatment of lingual tonsillitis. When conventional therapies fail. Int $J$ Med Robot 2017;13:e1763.

22. Friedman M, Ibrahim H, Bass L. Clinical staging for sleep-disordered breathing. Otolaryngol Head Neck Surg 2002;127:13-21.

23. Babademez MA, Gul F, Muz E, Muderris T, Kale H. Impact of partial and total tonsillectomy on adenoid regrowth. Laryngoscope 2017;127:753-756.

24. Wei L, Wang M, Hua N, Tong K, Zhai L, Wang Z. Regrowth of the adenoids after adenoidectomy down to the pharyngobasilar fascial surface. $J$ Laryngol Otol 2015;129:662-665.

25. Cammaroto G, Montevecchi F, D'Agostino G, et al. Tongue reduction for OSAHS: TORSs vs coblations, technologies vs techniques, apples vs oranges. Eur Arch Otorhinolaryngol 2017;274:637-645.

26. Nagel TH, Hinni ML, Hayden RE, Lott DG. Transoral laser microsurgery for the unknown primary: role for lingual tonsillectomy. Head Neck 2014; 36:942-946. 